\title{
Parâmetros Indicativos para Qualidade da Água em Nascentes com Diferentes Coberturas de Terra e Conservação da Vegetação Ciliar
}

\author{
Caio Vinicius Ferreira Marmontel ${ }^{1}$, Valdemir Antonio Rodrigues ${ }^{1}$
}

${ }^{1}$ Departamento de Recursos Naturais/Ciências Florestais, Universidade Estadual Paulista - UNESP, Botucatu/SP, Brasil

\begin{abstract}
RESUMO
O objetivo desta pesquisa foi avaliar e comparar a qualidade da água de quatro nascentes em diferentes coberturas de terra e conservação da vegetação no Córrego Pimenta. As coletas de água foram realizadas mensalmente durante um ano, avaliando dez parâmetros físico-químicos, sendo que os dados foram submetidos ao Teste de Tukey. A nascente um (N1) possui mata ciliar preservada; a nascente dois (N2) possui mata ciliar perturbada; a nascente três (N3) possui bambuzal e mata ciliar, e está degradada; a nascente quatro (N4) tem cobertura de pastagem e se encontra em degradação. As nascentes N1 e N2 tiveram os valores inferiores em temperatura, turbidez, cor, nitrato, nitrito e ferro, e valores próximos à neutralidade em $\mathrm{pH}$. A N3 mostrou os valores inferiores para condutividade elétrica, TDS, salinidade e $\mathrm{pH}$. As nascentes N1 e N2 indicaram que sete parâmetros foram melhores na qualidade da água, demonstrando que a mata ciliar auxilia em sua proteção qualitativa da água.
\end{abstract}

Palavras-chave: nascente, mata ciliar, parâmetros físico-químicos, pastagem.

\section{Indicative Parameters for Water Quality in Water Springs with Different Land Cover and Conservation of Riparian Vegetation}

\begin{abstract}
The objective of this study was to evaluate and compare the water quality of four water springs located in different land cover types and conservation of vegetation along the Pimenta stream. Water sampling was conducted monthly for one year. Ten physicochemical parameters were evaluated and data were submitted to the Tukey test. Water spring number one (N1) is located in an area of preserved riparian forest; water spring number two (N2) is located in an area of disturbed riparian forest; water spring number three (N3) is in an area of disturbed bamboo and riparian forest; and water spring number four (N4) is in a degrading area of pasture cover. N1 and N2 showed lower values for the parameters of temperature, turbidity, color, nitrate, nitrite and iron, and $\mathrm{pH}$ values close to neutral. $\mathrm{N} 3$ showed lower values for electrical conductivity, total dissolved solids (TDS), salinity and $\mathrm{pH}$. The results found for N1 and N2 indicate that seven parameters were higher regarding water quality, which demonstrates that native vegetation can help protect water quality.
\end{abstract}

Keywords: water springs, riparian forest, physicochemical parameters, pasture. 


\section{INTRODUÇÃO}

As matas ciliares exercem funções hidrológicas e ecológicas de proteção aos solos e aos recursos hídricos, por meio de: manutenção da qualidade da água; regularização dos cursos d'água; conservação na biodiversidade (Rodrigues, 2004); alternativas econômicas de exploração sustentável da biota; educação ambiental; pesquisa científica; paisagem de beleza cênica; turismo, e até a contribuição para a redução do efeito estufa, através da captura do carbono atmosférico (Braga, 2005).

A mata ciliar também atua na modificação dos processos químicos e biológicos, mudando a composição química dos componentes. Transforma, por exemplo, os resíduos de pesticidas transportados pelo escoamento em componentes não tóxicos por decomposição microbiológica, oxidação, redução, hidrólise, radiação solar e outras ações que ocorrem no piso florestal (Simões, 2001). A vegetação ciliar, em uma bacia, reduz em $38 \%$ a concentração de nitrogênio; em $94 \%$, o fosfato; $42 \%$, o fósforo dissolvido; $21 \%$, de alumínio total infiltrável, e $54 \%$ de ferro, que chegam ao curso d'água (Emmett et al., 1994).

As nascentes perdem as capacidades quantitativa e qualitativa da água, quando é alterada, por ações antrópicas, em ambientes de contribuição natural de infiltração em seu entorno e na área de recarga do lençol freático, comprometendo seu reabastecimento e sua produção de água (Rodrigues, 2006). A degradação das matas ciliares e sua substituição por atividades agropecuárias e expansão urbana vêm causando alterações na qualidade das águas e afetando o abastecimento público (Davide et al., 2002; Pinto, 2003; Grossi, 2006), necessitando de ajustes no planejamento e na gestão dos recursos hídricos (Iost, 2008).

Para uma gestão adequada dos recursos hídricos, o primeiro passo é o monitoramento da qualidade da água, a fim de caracterizar aspectos físico-químicos que permitem diagnosticar as mudanças ocasionadas por ações antrópicas ou naturais, no uso e na ocupação da terra (Queiroz et al., 2010). O monitoramento, em uma microbacia hidrográfica, da qualidade da água segundo os parâmetros físico-químicos é fundamental, para que, a partir das informações levantadas, seja possível um melhor entendimento das verdadeiras influências de cada processo de degradação (Silva, 2009).

O objetivo desta pesquisa foi avaliar e comparar a qualidade da água de quatro nascentes no Córrego Pimenta, em diferentes coberturas da terra e conservação da vegetação ciliar.

\section{MATERIAL E MÉTODOS}

\subsection{Caracterização da área}

O trabalho foi desenvolvido na sub-bacia do Córrego Pimenta, afluente do Rio Paraíso, na Fazenda Experimental São Manuel, pertencente à UNESP. Está situada entre as coordenadas geográficas de $22^{\circ} 46^{\prime} 07^{\prime \prime} \mathrm{S}$ a $22^{\circ} 46^{\prime} 57^{\prime \prime} \mathrm{S}, 48^{\circ} 33^{\prime} 49^{\prime \prime} \mathrm{W}$ a $48^{\circ} 33^{\prime} 59^{\prime \prime} \mathrm{W}$, e altitude média de 779 metros.

O clima da região de São Manuel, conforme Köppen (1948), é do tipo Cwa, clima temperado quente (mesotérmico). Os maiores índices hídricos (período de chuva) são registrados nas estações primavera-verão e as deficiências hídricas (período de seca), nas estações outono-inverno (CIIAGRO, 2013).

O local é constituído de sedimentos modernos, com pontos de afloramento do Arenito Bauru, formando pequenas elevações. As várzeas dos cursos de água têm seus terrenos aluviais formados por sedimentos arenosos (Lima, 2003). O solo é classificado como Latossolo Vermelho-Escuro, fase arenosa, denominado, segundo EMBRAPA (2006), como Latossolo Vermelho Distrófico. São solos em avançado estágio de intemperização, muito evoluídos, como resultado de enérgicas transformações no material constitutivo.

Para caracterizar o grau de conservação da vegetação ciliar das nascentes, foi adotado o procedimento descrito por Pinto (2003), medindo a vegetação nos quatro quadrantes da nascente em seu entorno, sendo que a margem direita e a esquerda foram orientadas pelo sentido do escoamento do leito do curso principal. Em seguida, as nascentes foram classificadas em relação à conservação da vegetação ciliar no seu entorno, ou seja, nascente preservada, perturbada ou degradada. Quando apresenta pelo menos 50 metros de vegetação natural ao seu redor, previstos na Lei n. ${ }^{\circ}$ 12.651/2012 (Brasil, 2012), e sem 
sinais de perturbação ou degradação, é denominada preservada. A nascente que não apresenta 50 metros de vegetação natural ao seu redor, previstos na referida Lei, mas se encontra em bom estado de conservação, mesmo sendo ocupada, em parte por pastagem ou agricultura, é classificada como perturbada. A nascente que se encontra com elevado grau de perturbação, solo compactado, vegetação escassa e com erosão ou voçoroca é classificada como degradada.

O material cartográfico usado foram as cartas do Instituto Geográfico e Cartográfico (IGC) na escala 1:10.000, com equidistância vertical de $5 \mathrm{~m}$, da área de estudo; em seguida, foi gerado o plano de informação da rede de drenagens. Para a elaboração do mapa de uso e cobertura da terra, foi utilizada uma Imagem do Google Earth (versão 7.1.2.2041), com uma resolução espacial de 10 metros, operando na APP em quatro bandas espectrais, sendo em verde, laranja, salmão e azul. Todo o banco de dados gerados foi utilizado no Sistema UTM (Universal Transverso de Mercator) e no datum SISRGAS 2000.

\subsection{Coleta de amostras da água}

As coletas da água foram amostradas nas quatro nascentes com quatro pontos cada, sendo o primeiro na nascente e os outros a $10 \mathrm{~m}, 30 \mathrm{~m}$ e $50 \mathrm{~m}$ na mesma direção longitudinal. As amostragens de águas superficiais foram efetuadas no período de um ano, entre agosto de 2012 e julho de 2013, distribuídas em coletas mensais, tendo sido realizando todo o procedimento de acordo com as técnicas descritas por APHA et al. (1992).

A temperatura $\left({ }^{\circ} \mathrm{C}\right)$ e o $\mathrm{pH}$ da água foram determinados pela leitura do phmetro (Modelo $\mathrm{PH}$ 100 - Extech); para determinar a condutividade elétrica $\left(\mu \mathrm{S} . \mathrm{cm}^{-1}\right)$, o TDS $\left(\mathrm{mg} . \mathrm{L}^{-1}\right)$ e a salinidade (mg. $\mathrm{L}^{-1}$ ), foi utilizado o condutivímetro portátil (Modelo EC 400 - Extech), sendo a análise dessas variáveis realizada no próprio local de cada ponto da nascente. As análises de turbidez (FAU), nitrato (mg.L $\mathrm{L}^{-1}$ ), nitrito (mg.L $\mathrm{L}^{-1}$ ) e ferro (mg.L $\mathrm{L}^{-1}$ ) foram determinadas com uso do espectrofotômetro digital (Modelo Hach DR2010), e o parâmetro cor (mg. $\mathrm{L}^{-1}$. $\mathrm{Pt}$ ), pelo colorímetro (Modelo 611-A Aqua-Tester). As análises foram realizadas no Laboratório de Qualidade de Água no Departamento de Engenharia Rural no Câmpus de Botucatu - FCA/UNESP.

\subsection{Análise dos dados}

A análise exploratória dos resultados de caráter qualitativo foi constituída dos gráficos para a série temporal de um ano de cada parâmetro, plotados em planilha eletrônica do Microsoft Office/Excel 2007. Os dados foram submetidos à análise de variância e, quando o valor do teste $\mathrm{F}$ indicou efeito significativo, foi utilizado o teste de Tukey $(p<0,05)$, sendo tal análise realizada no programa de estatística ASSISTAT (Silva \& Azevedo, 2002).

\section{RESULTADOS E DISCUSSÃO}

\subsection{Caracterização das nascentes}

O Córrego Pimenta tem uma área de preservação permanente (APP) com 22,8 ha, e a cobertura do solo presente nesta é a vegetação nativa, a pastagem, o bambuzal e o povoamento de Eucalyptus sp. (Figura 1).

A nascente $\mathrm{N} 1$ se encontra preservada em um remanescente florestal, com raio de 80 metros de APP no seu entorno, em condições de conservação previstas no código florestal. A vegetação tem características fisionômicas da Floresta Estacional Semidecidual e do Cerrado, devido a estar em uma região que é de transição entre essas formações. A nascente N2 também apresenta cobertura de mata ciliar de sucessão secundária, após sofrer queimadas 40 anos atrás. A $\mathrm{N} 2$ se encontra em estado de perturbação, no mesmo remanescente, mas não apresenta raio mínimo de mata ciliar no seu entorno.

A nascente $\mathrm{N} 3$ está degradada, possui cobertura de bambuzal (Bambusa sp.), que foi inserida para controle de erosão na cabeceira do córrego, seguida de uma faixa estreita de mata ciliar em estágio inicial, menor que os 50 metros previstos em lei, além de haver pastagem no raio da nascente. Há 50 anos ao redor da N3, eram despejados esgotos domésticos tratados e não tratados. A nascente N4 está degradada e predomina a pastagem com (Brachiaria sp.), não apresentando condições adequadas previstas no código florestal. Ao redor da nascente, na época das chuvas, deságuam no curso d'água produtos químicos e fertilizantes utilizados na pastagem, contribuindo para a degradação.

A presença de taboa [Thyphadomingensis (Pers.)] na N4 ocasiona o aumento de restos vegetais, 


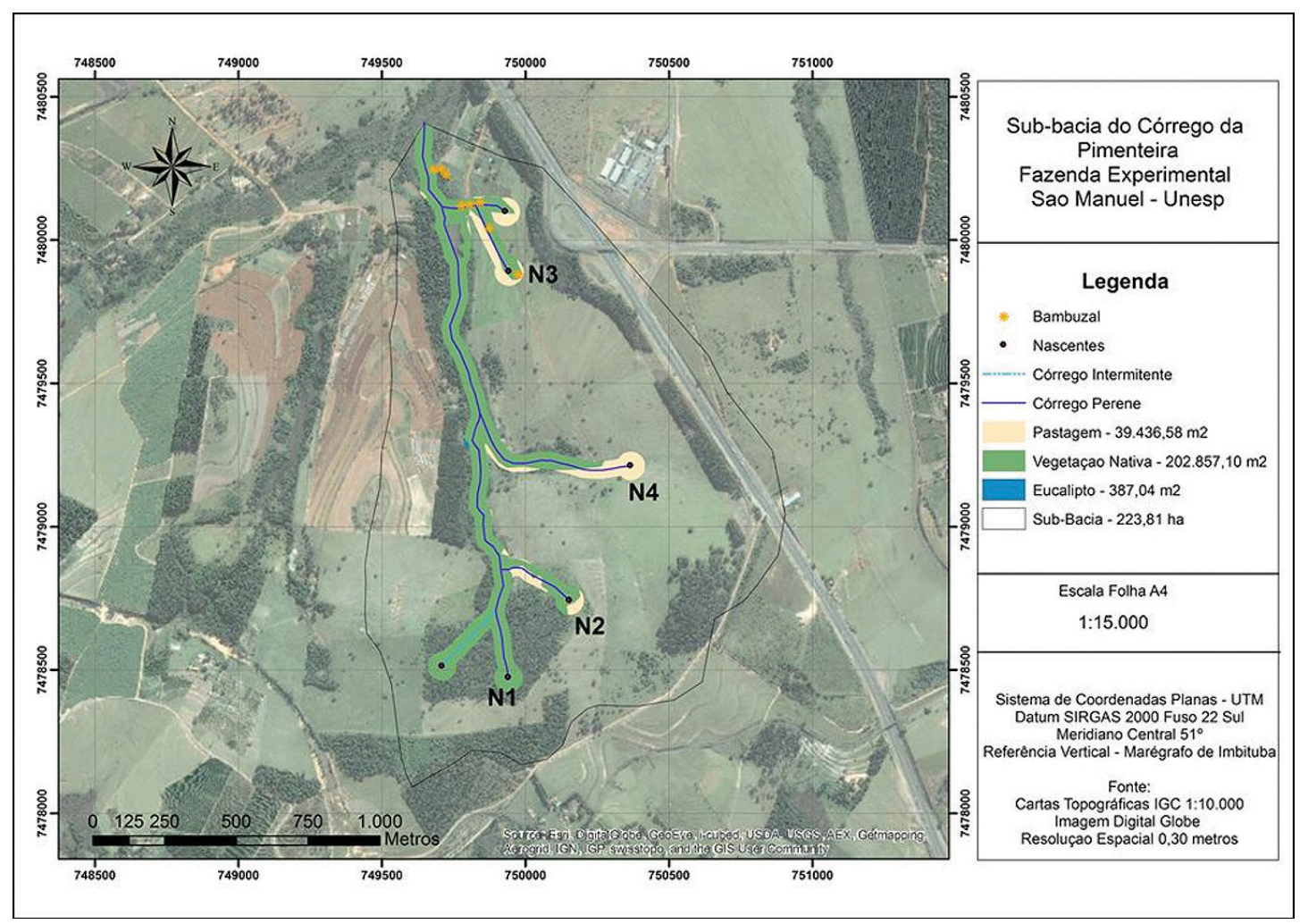

Figura 1. Mapa dos diferentes tipos de cobertura da terra na sub-bacia do Córrego Pimenta e a localização das quatro nascentes estudadas na Fazenda Experimental de São Manuel - FCA/UNESP.

Figure 1. Map of the different types of land cover on creek Pimenta in the sub-basin and the location of the four sources studied at the Experimental Farm of São Manuel -FCA/UNESP.

aumentando o teor de matéria orgânica da água e intensificando o desenvolvimento de microrganismos. Há também, na nascente, uma faixa de contorno não vegetada utilizada como estrada, deixando mais desprotegida a área que deveria ser composta pela mata ciliar.

\subsection{Parâmetros qualitativos da água}

Nas Figuras 2 a 11, observa-se que a temperatura, a turbidez, a cor, o nitrato, o nitrito e o ferro tiveram os menores valores para as nascentes $\mathrm{N} 1 \mathrm{e} \mathrm{N} 2$, enquanto a condutividade elétrica, o TDS, a salinidade e o pH tiveram os menores valores para $\mathrm{N} 3$.

A temperatura da $\mathrm{N} 1$ teve as menores médias durante o período avaliado, refletindo as condições

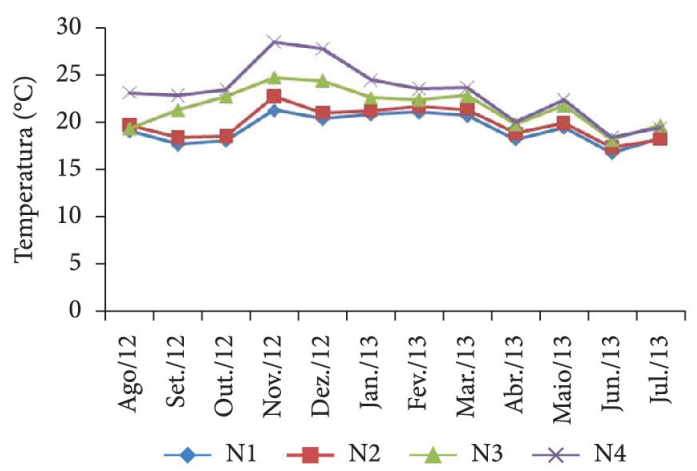

Figura 2. Valores médios mensais da temperatura da água durante um ano, em quatro nascentes no Córrego Pimenta, São Manuel-SP.

Figure 2. Monthly average values water temperature for one year in four sources in the creek Pimenta, San Manuel/SP. 


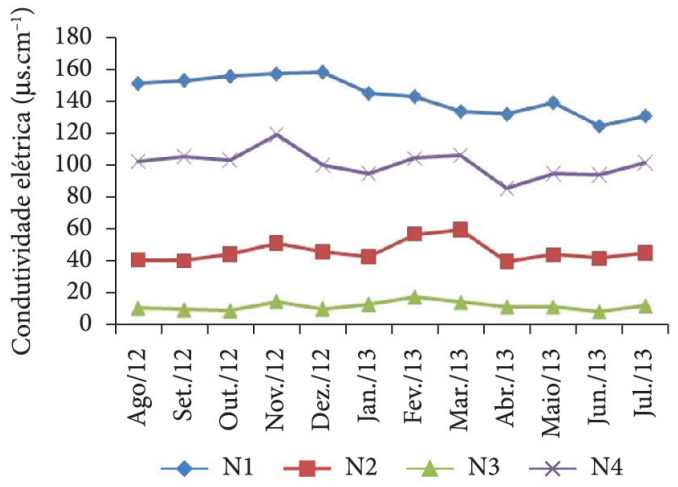

Figura 3. Valores médios mensais da condutividade elétrica da água durante um ano, em quatro nascentes no Córrego Pimenta, São Manuel-SP.

Figure 3. Monthly average values water electrical conductivity for one year in four sources in the creek Pimenta, San Manuel/SP.

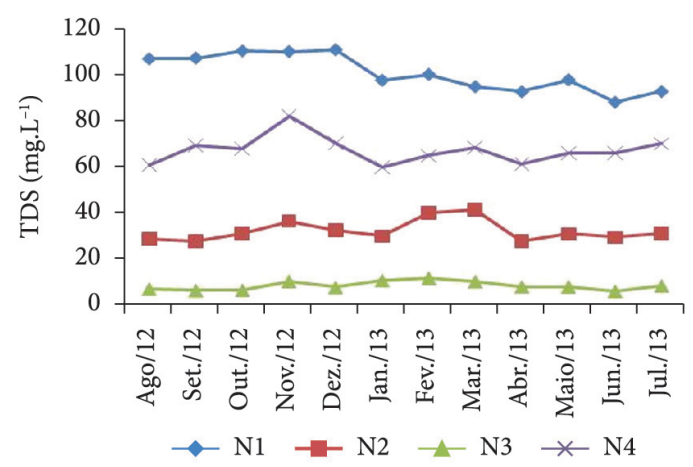

Figura 4. Valores médios mensais do TDS da água durante um ano, em quatro nascentes no Córrego Pimenta, São Manuel-SP.

Figure 4. Monthly average values water TDS for one year in four sources in the creek Pimenta, San Manuel/SP.

de sombreamento da mata ciliar no entorno das duas nascentes. A condutividade elétrica, o TDS e a salinidade da água tiveram comportamentos semelhantes entre as nascentes, sendo que a N3 e a $\mathrm{N} 2$ tiveram valores inferiores, enquanto a $\mathrm{N} 1$ e a $\mathrm{N} 4$ tiveram os maiores valores, durante o monitoramento.

$\mathrm{O} \mathrm{pH}$ das nascentes $\mathrm{N} 1$ e $\mathrm{N} 2$ foi próximo à neutralidade, enquanto a $\mathrm{N} 3$ teve o $\mathrm{pH}$ mais baixo. Os parâmetros de turbidez, cor, nitrato, nitrito e ferro em N1 e N2 apresentaram valores baixos, além da estabilidade durante o período avaliado, enquanto que N3 e N4 tiveram os níveis mais elevados, além de variações durante o monitoramento. A estabilidade dos valores proporciona ao ambiente um

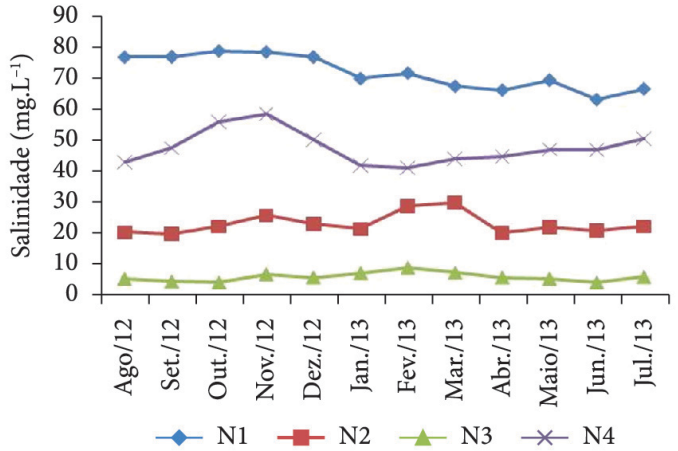

Figura 5. Valores médios mensais da salinidade da água durante um ano, em quatro nascentes no Córrego Pimenta, São Manuel-SP.

Figure 5. Monthly average values water salinity for one year in four sources in the creek Pimenta, San Manuel/ SP.

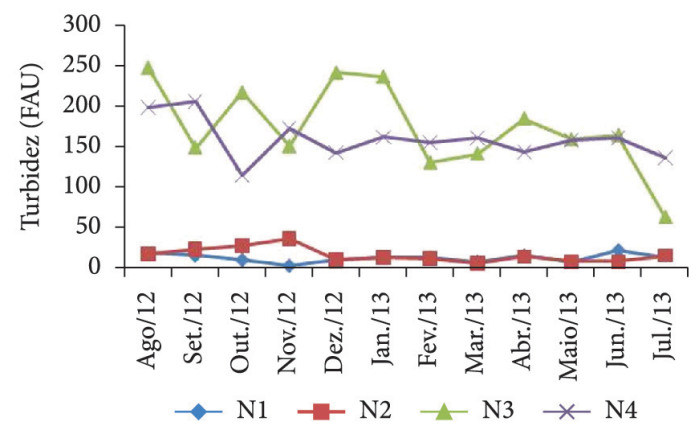

Figura 6. Valores médios mensais da turbidez da água durante um ano, em quatro nascentes no Córrego Pimenta, São Manuel-SP.

Figure 6. Monthly average values water turbidity for one year in four sources in the creek Pimenta, San Manuel/SP

desenvolvimento adequado, ao contrário de valores com grandes variações, que prejudicam o equilíbrio do recurso hídrico.

Arcova \& Cicco (1999), Donadio et al. (2005) e Silva (2009) constataram menores temperaturas durante os estudos para microbacias florestadas quando comparadas com atividades agrícolas. Valores de $\mathrm{pH}$ próximos à neutralidade foram semelhantes ao estudo de Donadio et al. (2005), com uso do solo de vegetação natural. De acordo com Silva (2007), quando há pouca alteração nos valores de $\mathrm{pH}$, isso contribui para a manutenção de seres vivos presentes nos locais. Variações espaciais de parâmetros físico-químicos indicam que o ambiente aquático é 


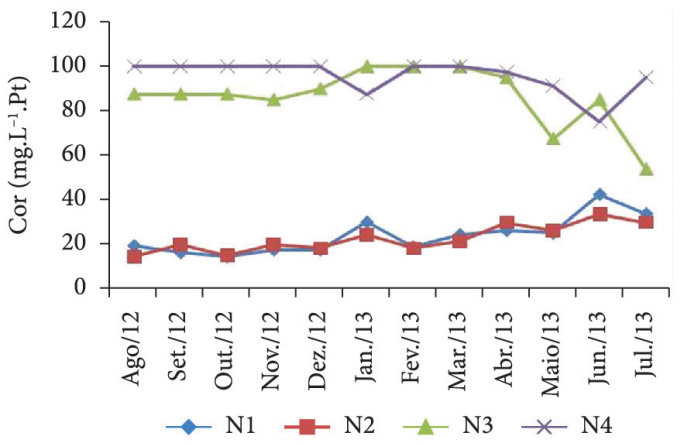

Figura 7. Valores médios mensais da cor da água durante um ano, em quatro nascentes no Córrego Pimenta, São Manuel-SP.

Figure 7. Monthly average values water color for one year in four sources in the creek Pimenta, San Manuel/ SP.

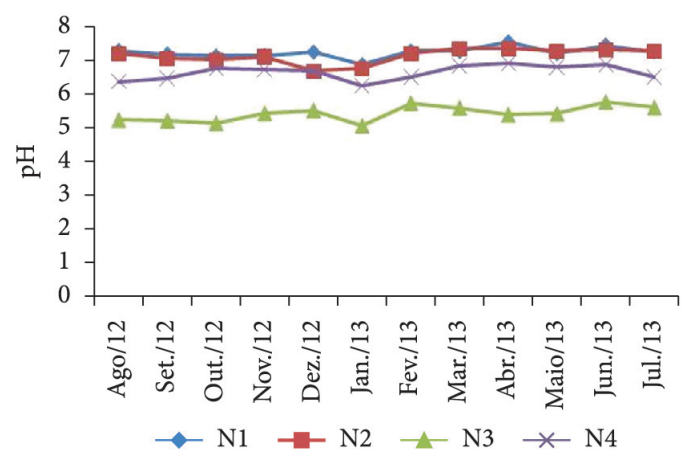

Figura 8. Valores médios mensais do $\mathrm{pH}$ da água durante um ano, em quatro nascentes no Córrego Pimenta, São Manuel-SP.

Figure 8. Monthly average values water $\mathrm{pH}$ for one year in four sources in the creek Pimenta, San Manuel/SP.

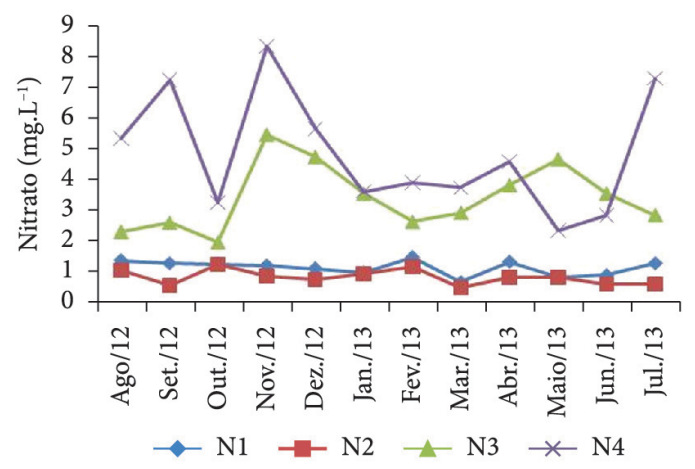

Figura 9. Valores médios mensais do nitrato da água durante um ano, em quatro nascentes no Córrego Pimenta, São Manuel-SP.

Figure 9. Monthly average values water nitrate for one year in four sources in the creek Pimenta, San Manuel/SP.

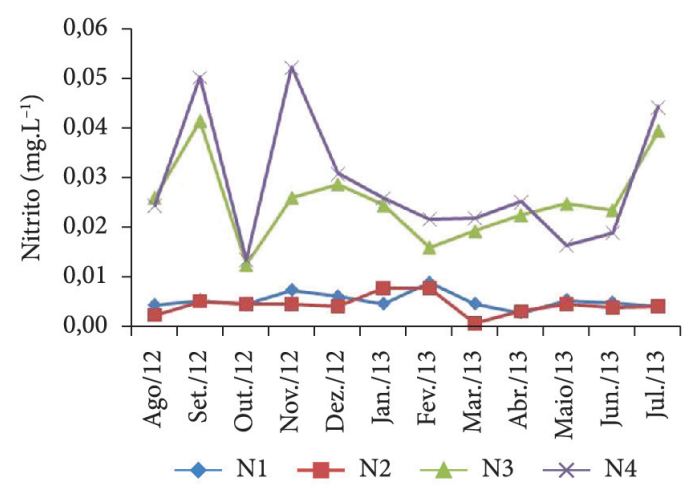

Figura 10. Valores médios mensais do nitrito da água durante um ano, em quatro nascentes no Córrego Pimenta, São Manuel-SP.

Figure 10. Monthly average values water nitrite for one year in four sources in the creek Pimenta, San Manuel/ SP.

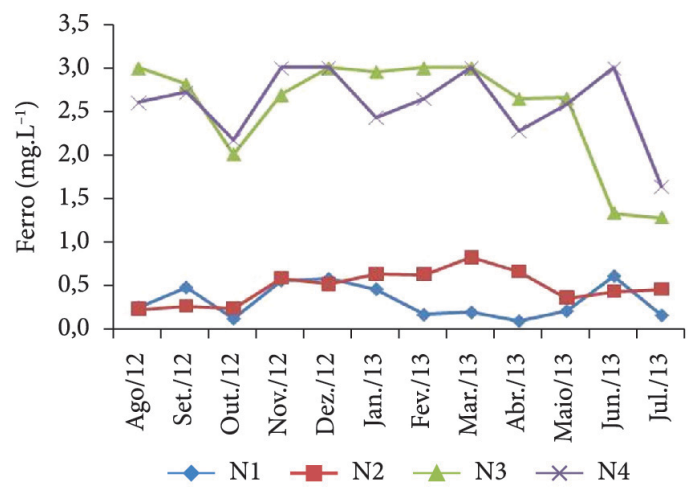

Figura 11. Valores médios mensais do ferro da água durante um ano em quatro nascentes no Córrego Pimenta, São Manuel-SP.

Figure 11. Monthly average values water iron for one year in four sources in the creek Pimenta, San Manuel/ SP.

sensível às modificações no ambiente, aumentando as concentrações de materiais em suspensão na porção do córrego em que a vegetação foi retirada (Bleich et al., 2009).

Primavesi et al. (2002), Mosca (2003) e Donadio et al. (2005) apontaram valores maiores para turbidez em microbacias com uso agrícola do que em áreas florestadas, evidenciando, assim, a função da mata ciliar na contenção de sólidos que poderiam atingir a água. Arcova \& Cicco (1999) e Donadio et al. (2005) também verificaram menores valores do parâmetro cor da água na mata ciliar em comparação a outros tipos de uso do solo. 
No período de seca, a temperatura foi maior para $\mathrm{N} 4$, diferindo de N1 e N2. No período de chuva, a N4 diferiu para as outras três nascentes estudadas, apresentando a maior temperatura. Tanto no período seco como no período chuvoso, $\mathrm{N} 1$ e N2 não diferiram estatisticamente quanto à temperatura (Tabela 1 ).

As nascentes $\mathrm{N} 1$ e N2 tiveram as menores médias de temperatura, mostrando a importância da presença da vegetação ciliar. A N4, desprotegida da vegetação ciliar, e a N3, com uma pequena faixa de vegetação, ficam bastante expostas à radiação solar direta; consequentemente, elevam-se as temperaturas. A presença da mata ciliar é a maneira mais efetiva de prevenir aumentos da temperatura da água, oferecendo maior proteção, devido à menor exposição dos canais à luz solar direta (Arcova \& Cicco, 1999).

Tabela 1. Efeito das nascentes com diferentes coberturas de terra e a conservação da vegetação ciliar de dez parâmetros físico-químicos da água, nos períodos de seca e de chuva, no Córrego Pimenta, São Manuel-SP.

Table 1. Effect of sources with different coverings of soil and status conservation of the vegetation on ten physicalchemical parameters of water in periods of drought and rain in the creek Pimenta, São Manuel/SP.

\begin{tabular}{|c|c|c|c|c|c|}
\hline \multirow{2}{*}{ Nascentes } & \multicolumn{2}{|c|}{$\mathrm{T}\left({ }^{\circ} \mathrm{C}\right)$} & \multirow{2}{*}{ Nascentes } & \multicolumn{2}{|c|}{ Cor (mg.L.'-1.Pt) } \\
\hline & Ps & Pc & & Ps & Pc \\
\hline $\mathrm{N} 1$ & $18,2 \mathrm{c}$ & $20,4 \mathrm{c}$ & $\mathrm{N} 1$ & $27,2 \mathrm{~b}$ & $20,4 \mathrm{~b}$ \\
\hline N2 & $18,7 \mathrm{bc}$ & $21,1 \mathrm{c}$ & $\mathrm{N} 2$ & $25,8 \mathrm{~b}$ & $19,5 \mathrm{~b}$ \\
\hline N3 & $20 \mathrm{ab}$ & $23,3 \mathrm{~b}$ & N3 & $79,4 \mathrm{a}$ & $93,8 \mathrm{a}$ \\
\hline N4 & $21 \mathrm{a}$ & $25,2 \mathrm{a}$ & N4 & $93,1 \mathrm{a}$ & $97,9 \mathrm{a}$ \\
\hline CV (\%) & 7,1 & 6,9 & $\mathrm{CV}(\%)$ & 19,3 & 9,4 \\
\hline \multirow{2}{*}{ Nascentes } & \multicolumn{2}{|c|}{ Cond. $\left(\mu \mathrm{S} . \mathrm{cm}^{-1}\right)$} & \multirow{2}{*}{ Nascentes } & \multicolumn{2}{|c|}{$\mathrm{pH}$} \\
\hline & Ps & Pc & & Ps & Pc \\
\hline $\mathrm{N} 1$ & $139 \mathrm{a}$ & $149 \mathrm{a}$ & N1 & $7,4 \mathrm{a}$ & $7,2 \mathrm{a}$ \\
\hline $\mathrm{N} 2$ & $41,8 \mathrm{c}$ & $50,2 \mathrm{c}$ & $\mathrm{N} 2$ & $7,3 \mathrm{a}$ & $7 \mathrm{a}$ \\
\hline N3 & $10,7 \mathrm{~d}$ & $13,2 \mathrm{~d}$ & N3 & $5,5 \mathrm{c}$ & $5,4 \mathrm{c}$ \\
\hline $\mathrm{N} 4$ & $97,3 \mathrm{~b}$ & $105 \mathrm{~b}$ & N4 & $6,7 \mathrm{~b}$ & $6,7 \mathrm{~b}$ \\
\hline CV (\%) & 9,6 & 9,4 & CV (\%) & 2,8 & 3,4 \\
\hline \multirow{2}{*}{ Nascentes } & \multicolumn{2}{|c|}{ TDS (mg.L -1 $\left.^{-1}\right)$} & \multirow{2}{*}{ Nascentes } & \multicolumn{2}{|c|}{$\mathrm{NO}_{3}^{-}-\mathrm{N}\left(\mathrm{mg} \cdot \mathrm{L}^{-1}\right)$} \\
\hline & Ps & Pc & & Ps & Pc \\
\hline N1 & $97,5 \mathrm{a}$ & $104 \mathrm{a}$ & N1 & $1,2 \mathrm{~b}$ & $1,1 \mathrm{~b}$ \\
\hline N2 & $29 c$ & $35 c$ & $\mathrm{~N} 2$ & $0,7 \mathrm{~b}$ & $0,9 \mathrm{~b}$ \\
\hline N3 & $7,2 \mathrm{~d}$ & $9,4 \mathrm{~d}$ & N3 & $3,3 \mathrm{a}$ & $3,5 \mathrm{a}$ \\
\hline N4 & $65,4 \mathrm{~b}$ & $68,7 \mathrm{~b}$ & $\mathrm{~N} 4$ & $5 \mathrm{a}$ & $4,8 \mathrm{a}$ \\
\hline CV (\%) & 9,2 & 10,8 & CV (\%) & 46,4 & 47,3 \\
\hline \multirow{2}{*}{ Nascentes } & \multicolumn{2}{|c|}{ Sali. (mg. $\left.\mathrm{L}^{-1}\right)$} & \multirow{2}{*}{ Nascentes } & \multicolumn{2}{|c|}{$\mathrm{NO}_{2}{ }^{-}-\mathrm{N}\left(\mathrm{mg} \cdot \mathrm{L}^{-1}\right)$} \\
\hline & Ps & Pc & & Ps & Pc \\
\hline N1 & $69,7 \mathrm{a}$ & $73,7 \mathrm{a}$ & N1 & $0 \mathrm{~b}$ & $0,01 \mathrm{~b}$ \\
\hline $\mathrm{N} 2$ & $20,7 \mathrm{c}$ & $25 c$ & $\mathrm{~N} 2$ & $0 \mathrm{~b}$ & $0,01 \mathrm{~b}$ \\
\hline N3 & $5,1 \mathrm{~d}$ & $6,6 \mathrm{~d}$ & N3 & $0,03 \mathrm{a}$ & $0,02 \mathrm{a}$ \\
\hline N4 & $46,6 \mathrm{~b}$ & $48,5 \mathrm{~b}$ & N4 & $0,03 \mathrm{a}$ & $0,03 \mathrm{a}$ \\
\hline CV (\%) & 9,1 & 12,5 & CV (\%) & 48,5 & 49,8 \\
\hline \multirow{2}{*}{ Nascentes } & \multicolumn{2}{|c|}{ Turb. (FAU) } & \multirow{2}{*}{ Nascentes } & \multicolumn{2}{|c|}{$\mathrm{Fe}\left(\mathrm{mg} \cdot \mathrm{L}^{-1}\right)$} \\
\hline & Ps & Pc & & Ps & Pc \\
\hline N1 & $15,6 \mathrm{~b}$ & $9,7 \mathrm{~b}$ & N1 & $0,3 \mathrm{~b}$ & $0,4 \mathrm{~b}$ \\
\hline N2 & $14,3 \mathrm{~b}$ & $17,5 \mathrm{~b}$ & N2 & $0,4 \mathrm{~b}$ & $0,6 \mathrm{~b}$ \\
\hline $\mathrm{N} 3$ & $161 \mathrm{a}$ & $186 \mathrm{a}$ & $\mathrm{N} 3$ & $2,3 \mathrm{a}$ & $2,8 \mathrm{a}$ \\
\hline N4 & $167 \mathrm{a}$ & $152 \mathrm{a}$ & N4 & $2,5 \mathrm{a}$ & $2,7 \mathrm{a}$ \\
\hline CV (\%) & 37,2 & 30,8 & CV (\%) & 34,7 & 19,2 \\
\hline
\end{tabular}

Ps = período de seca; $\mathrm{Pc}=$ período de chuva; $\mathrm{T}=$ temperatura $\left({ }^{\circ} \mathrm{C}\right) ;$ Cond. = condutividade elétrica $\left(\mu \mathrm{S} . \mathrm{cm}^{-1}\right) ; \mathrm{TDS}=$ sólidos dissolvidos totais $\left(\mathrm{mg} \cdot \mathrm{L}^{-1}\right) ;$ Sali. $=$ salinidade $\left(\mathrm{mg} \cdot \mathrm{L}^{-1}\right) ;$ Turb. $=$ turbidez $(\mathrm{FAU}) ; \operatorname{cor}\left(\mathrm{mg} \cdot \mathrm{L}^{-1} \cdot \mathrm{Pt}\right) ; \mathrm{ph}=$ potencial hidrogeniônico; $\mathrm{NO}_{3}$ $-\mathrm{N}=$ nitrato $\left(\mathrm{mg} . \mathrm{L}^{-1}\right) ; \mathrm{NO}_{2}^{-}-\mathrm{N}=$ nitrito $\left(\mathrm{mg} \cdot \mathrm{L}^{-1}\right) ; \mathrm{Fe}=$ ferro $\left(\mathrm{mg} \cdot \mathrm{L}^{-1}\right) ; \mathrm{CV}=$ coeficiente de variação (\%).

Valores médios seguidos por letras minúsculas iguais na linha não diferem estatisticamente pelo Teste de Tukey $(p<0,05)$. 
A condutividade elétrica, o TDS e a salinidade, nos períodos de seca e de chuva, tiveram diferenças significativas entre as quatro nascentes estudadas, se diferenciando estatisticamente entre estas. Os valores altos de condutividade elétrica da N1, mesmo com a presença da vegetação ciliar, demonstram que há outros fatores que também afetam a qualidade da água, que não a simples existência de mata ciliar protegendo a APP; por sua vez, a N2, também com a presença da vegetação ciliar, apresentou tais valores baixos, mesmo sendo constituída por solos em avançado estágio de intemperização. A N4 pode ter sido influenciada pelas ações antrópicas, ou seja, utilização de fertilizantes e defensivos agrícolas na pastagem, além de resíduos oriundos dos animais de atividade intensiva de pecuária que chegam ao curso d'água. A elevada concentração de $\mathrm{N} 1$ e N4, e os valores baixos de $\mathrm{N} 2$ e N3 mostram que a cobertura de terra e a conservação da vegetação têm influência nos valores da condutividade elétrica, porém outros fatores não detectáveis nesse estudo podem influenciar também esse parâmetro.

De acordo com Arcova et al. (1998) e Arcova \& Cicco (1999), os valores médios mensais de condutividade elétrica das microbacias florestadas foram inferiores a $20 \mu \mathrm{s} . \mathrm{cm}^{-1}$, sendo o fator determinante a geologia do local, constituída por rochas resistentes ao intemperismo, como granitos e gnaisses. De acordo com Arcova \& Cicco (1999), não foi possível constatar qualquer influência do uso do solo sobre esse parâmetro da água. Fernandes et al. (2011) observaram que a pastagem bem manejada na microbacia oferece benefícios de proteção ao solo e não aumenta a quantidade de sólidos totais na água.

A turbidez e a cor da água, tanto no período seco quanto no período chuvoso, tiveram diferenças significativas entre as nascentes, sendo que N1 diferenciou de $\mathrm{N} 3$ e N4, e foi estatisticamente semelhante à N2. As nascentes N1 e N2, com maior parte de cobertura de vegetação ciliar, mostram que tal condição contribuiu para obter valores mais baixos. Um dos fatores que acarretaram valores altos de turbidez nas nascentes N3 e N4 pode ser o fato de o solo estar desprotegido pela mata ciliar. Nesse quadro de não proteção, pode ocorrer o transporte de material particulado em suspensão no curso d'água por estar mais suscetível à erosão, além da presença de uma estrada de terra situada próximo ao curso d’água principal da N4. Na N3, houve o despejo doméstico em sua cabeceira, sendo que este fato pode ter colaborado com a elevação da turbidez, ocorrendo, dessa forma, pelo aumento da presença de sólidos em suspensão, e interferindo no grau de penetração da luz através da água.

A elevação dos valores de turbidez por conta da escassez da mata ciliar e a presença de uma estrada foram observadas também no estudo de Arcova \& Cicco (1999), em uma microbacia de uso do solo com agricultura. Medeiros et al. (2013) concluíram que a qualidade das águas das bacias se desqualificou devido aos processos erosivos que carreiam sedimentos ao canal fluvial, elevando a turbidez, sendo tais problemas solucionados pelo ordenamento do uso, da ocupação e do manejo da terra. Arcova \& Cicco (1999) observaram valores superiores da cor e evidenciaram que os sedimentos em suspensão e o carreamento de material para o córrego interferiram na coloração das águas.

$\mathrm{O} \mathrm{pH}$ da água, tanto no período de seca quanto no período de chuva, teve variações significativas entre as nascentes: a N1 se diferenciou de $\mathrm{N} 3$ e N4, e foi estatisticamente semelhante à N2, enquanto a N3 se diferenciou de N4. Nas nascentes N1 e N2, mostrou-se que a presença da mata ciliar (preservada e perturbada) influenciou no equilíbrio dos valores de $\mathrm{pH}$. A N3 apresentou os valores mais baixos, ocasionados pela sua utilização como despejo doméstico na cabeceira da nascente, aumentando as concentrações de ácidos orgânicos dissolvidos de origem autóctone. Derísio (2000) afirma que maiores alterações referentes ao $\mathrm{pH}$ são provocadas por despejos industriais e domésticos.

O nitrato e o nitrito da água nos períodos de seca e de chuva tiveram variações significativas entre as nascentes, em que a N1 se diferenciou de N3 e N4, e foi estatisticamente semelhante à N2. Os valores apresentados de nitrato e nitrito da água nas duas nascentes - N1e N2 - reforçam que a presença da vegetação ciliar, preservada ou até mesmo perturbada, influencia concentrações mais baixas desses parâmetros. Por sua vez, a nascente com presença da pastagem (N4) e a nascente com bambuzal na cabeceira e com uma pequena faixa de vegetação ciliar (N3) mostraram que a ausência ou somente uma faixa da vegetação ciliar em estado degradado contribui para a elevação dos valores de nitrato e 
nitrito. Outro fator que pode ter contribuído para os valores mais altos de N3 e N4 é o uso de fertilizantes nitrogenados de forma inadequada, próximo às nascentes.

Geralmente, as concentrações de nitrato em águas com presença de vegetação ciliar são muitas baixas, por este ser utilizado em mecanismos bioquímicos de solos e plantas, e em processos de denitrificação (Resende, 2002). Segundo Barros (2004), com o emprego e o uso de fertilizantes e excrementos de animais, esse material pode ser carreado pelo escoamento superficial para os cursos d'água. Castro \& Mendonça (2004), Crepalli (2007) e Silva (2007) também registraram maiores quantidades de nitrato devido às práticas agrícolas e à exposição do solo por cobertura de pouca proteção efetiva, além da aplicação de fertilizantes. Analisando os impactos das atividades humanas em bacias reflorestadas, Castro \& Mendonça (2004) constataram uma melhoria na qualidade de água em relação à quantidade de nitrato e nitrito nas microbacias com cobertura vegetal densa e em fase de regeneração avançada.

O parâmetro ferro, tanto no período de seca quanto no período de chuva, teve variações significativas entre as nascentes: $\mathrm{N} 1$ se diferenciou de N3 e N4, e foi estatisticamente semelhante à N2. Os valores apresentados de ferro na água, nas duas nascentes (N1e N2), reforçam que a presença da vegetação ciliar, preservada ou até mesmo perturbada, constitui fatores que influenciam concentrações mais baixas desse parâmetro. Por sua vez, a nascente com presença da pastagem (N4) e a nascente com bambuzal na cabeceira e com uma pequena faixa de vegetação ciliar (N3) mostraram que a ausência ou somente uma faixa da vegetação ciliar em estado degradado contribui para a elevação dos valores de ferro.

Uma microbacia hidrográfica com floresta natural não perturbada possui as melhores condições, em relação à proteção dos recursos hídricos, e elevada qualidade para o abastecimento doméstico. Estas podem servir como modelo de monitoramento em comparação com outras microbacias impactadas (Arcova \& Cicco, 1999).

As nascentes N1 e N2 apresentaram melhores resultados em relação à qualidade da água e não significativos, entre estas, nos parâmetros de temperatura, turbidez, cor, $\mathrm{pH}$, nitrato, nitrito e ferro. As nascentes N3 e N4 tiveram piores resultados e não significativos, entre estas, nos parâmetros de temperatura, turbidez, cor, nitrato, nitrito e ferro. Nos parâmetros de condutividade elétrica, TDS e salinidade, os melhores resultados foram para a N3 e os piores resultados, para a N1. Deve-se salientar que a utilização da água como indicador qualitativo exige estudos complementares, que permitam verificar outros fatores que possam interferir na sua qualidade.

\section{CONCLUSÕES}

A cobertura da terra com mata ciliar nos estados preservada e perturbada, respectivamente $\mathrm{N} 1$ e N2, contribuiu para uma melhor qualidade da água nos parâmetros de temperatura, turbidez, cor, $\mathrm{pH}$, nitrato, nitrito e ferro, enquanto a cobertura de terra com bambuzal e uma faixa de mata ciliar em estado degradado contribuiu para uma melhor qualidade da água nos parâmetros de condutividade elétrica, TDS e salinidade.

\section{AGRADECIMENTOS}

À Coordenação de Aperfeiçoamento de Pessoas de Nível Superior (CAPES), pela concessão da Bolsa de Estudo; aos funcionários da Fazenda Experimental de São Manuel da UNESP-FCA Nilton, Laércio, Donizete, Daniel e Rocha, por todo apoio e auxílio em campo; ao Thiago (Magrelox) e aos estagiários Gleico, Rodolfo (Dorfera) e Paulo Murilo (Murilão), por toda ajuda na coleta de dados em campo e na atividade de laboratório; ao Pesquisador Dr. Valdir de Cicco (Instituto Florestal do Estado de São Paulo) e aos Professores Dr. Renato Farias do Valle Junior (Instituto Federal do Triângulo Mineiro) e Dr. Raimundo Leite Cruz (UNESP-FCA), pelas críticas construtivas que contribuíram para a melhoria da redação científica do artigo; ao Técnico José Israel Ramos (UNESP-FCA), pelo ensinamento das técnicas de análises da qualidade da água.

\section{STATUS DA SUBMISSÃO}

Recebido: 13 mar., 2014

Aceito: 10 set., 2014 
AUTOR(ES) PARA CORRESPONDÊNCIA

\section{Caio Vinicius Ferreira Marmontel}

Departamento de Recursos Naturais/Ciências

Florestais, Universidade Estadual Paulista -

UNESP, Rua José Barbosa de Barros, 1780, CEP

18610-307, Botucatu, SP, Brasil

e-mail: caioo_marmontel@hotmail.com

\section{REFERÊNCIAS}

American Public Health Association - APHA, American Water Works Association - AWWA, Water Environment Federation - WEF. Standard methods: for the examination of water and wastewater. 18th ed. Washington: APHA:AWWA:WEF; 1992.

Arcova FCS, Cesar SF, Cicco V. Qualidade da água em microbacias recoberta por floresta de Mata Atlântica, Cunha, SP. Revista do Instituto Florestal 1998; 10(2): 185-196.

Arcova FCS, Cicco V. Qualidade da água de microbacia com diferentes usos do solo na região de Cunha, Estado de São Paulo. Scientia Forestalis 1999; 56: 125-134.

Barros RC. Agricultura e sustentabilidade ambiental: a qualidade da água dos rios formadores da Bacia do rio Grande - Nova Friburgo/RJ [tese]. Rio de Janeiro: Universidade Federal do Rio de Janeiro; 2004.

Bleich ME, Silva CJ, Rossete AN. Variação temporal e espacial das características limnológicas de um ecossistema lótico no Cerrado do mato Grosso. Biotemas 2009; 22(2): 161-171.

Braga RAP. Avaliação dos instrumentos de Políticas Públicas na Conservação Integrada de Florestas e Águas, com Estudo de Caso na Bacia do Corumbataí - SP [tese]. São Carlos: Escola de Engenharia de São Carlos, Universidade de São Paulo; 2005.

Brasil. Lei no 12.651, de 25 de maio de 2012. Revoga a Lei $n^{\circ}$ 4.771, de 15 de setembro de 1965, que dispõe sobre a proteção da vegetação nativa. Diário Oficial da República Federativa do Brasil, Brasília, DF (2012 maio 28); Sec. 1: 1-8.

Castro CB, Mendonça ASF. Impactos de ações antrópicas em bacias de mananciais rurais de montanha sobre parâmetros de qualidade de água. Revista Brasileira de Recursos Hídricos 2004; 9(1): 17-26.

Centro Integrado de Informações Agrometeorológicas - CIIAGRO. Monitoramento climático [online]. São Paulo: IAC:APTA:SAB; 2013. [cited 2013 Ago 14]. Available from: http://www.ciiagro.sp.gov.br/ ciiagroonline/Listagens/MonClim/LMClimLocal.asp.

Crepalli MS. Qualidade da água do rio Cascavel [dissertação]. Cascavel: Centro de Ciências Exatas e Tecnológicas, Universidade Estadual do Oeste do Paraná; 2007.

Davide AC, Pinto LVA, Monnerat PF, Botelho SA. Nascente: o verdadeiro tesouro da propriedade rural -o que fazer para conservar as nascentes nas propriedades rurais. Lavras: UFLA; 2002.

Derísio JC. Introdução ao controle de poluição ambiental. 2nd ed. São Paulo: Signus; 2000

Donadio NMM, Galbiatti JA, Paula RC. Qualidade da água de nascentes com diferentes usos do solo na bacia hidrográfica do córrego Rico, São Paulo, Brasil. Engenharia Agrícola 2005; 25(1): 115-125. http://dx.doi. org/10.1590/S0100-69162005000100013.

Emmett BA, Hudson JA, Cowar DPA, Reynolds B. The impact of a riparian wetland on streamwater quality in a recently afforested upland catchment. Journal of Hydrology 1994; 162(3-4): 337-353. http://dx.doi. org/10.1016/0022-1694(94)90235-6.

Empresa Brasileira de Pesquisa Agropecuária Embrapa, Centro Nacional de Pesquisa de Solos. Sistema brasileiro de classificação de solos. 2nd ed. Rio de Janeiro: EMBRAPA-SPI; 2006.

Fernandes MM, Ceddia MB, Ramos GM, Gaspar A, Moura MR. Influência do uso do solo na qualidade de água da microbacia Glória, Macaé - RJ. Engenharia Ambiental 2011; 8(2): 105-116.

Grossi CH. Diagnóstico e monitoramento ambiental da microbacia hidrográfica do rio Queima-Pé, MT [tese]. Botucatu: Faculdade de Ciências Agronômicas, Universidade Estadual Paulista; 2006.

Iost C. Produção de sedimentos e qualidade da água de uma microbacia hidrográfica rural [dissertação]. Cascavel: Universidade Estadual do Oeste do Paraná; 2008.

Köppen W. Climatologia: com um estúdio de los climas de latierra. México: Fondo de Cultura Econômica; 1948.

Lima PRA. Balanço de espécies químicas em microbacias sob utilização agrícola - região de Botucatu/SP [tese]. Botucatu: Faculdade de Ciências Agronômicas, Universidade Estadual Paulista; 2003.

Medeiros RB, Pinto AL, Miguel AES. Implicações da vulnerabilidade ambiental na qualidade das águas superficiais da Bacia do Córrego das Antas, Tupi Paulista/SP. Fórum Ambiental da Alta Paulista 2013; 9(2): 34-50.

Mosca AAO. Caracterização hidrológica de duas microbacias visando a identificação de indicadores hidrológicos para o monitoramento ambiental do manejo de florestas plantadas [dissertação]. Piracicaba: Escola Superior de Agricultura "Luiz de Queiroz", Universidade de São Paulo; 2003.

Pinto LVA. Caracterização física da bacia do ribeirão Santa Cruz, Lavras, MG, e propostas de recuperação 
de suas nascentes [dissertação]. Lavras: Universidade Federal de Lavras; 2003.

Primavesi O, Freitas AR, Primavesi AC, Oliveira HT. Water quality of the Canchim's Creek watershed in São Carlos, SP, Brasil, occupied by beef and dairy cattle activities. Brazilian Archives of Biology and Technology 2002; 45(2): 209-218. http://dx.doi.org/10.1590/S151689132002000200013 .

Queiroz MMF, Iost C, Gomes SD, Vilas Boas MA. Influência do uso do solo na qualidade da água de uma microbacia hidrográfica rural. Revista Verde de Agroecologia e Desenvolvimento Sustentável 2010; 5(4): 200-210.

Resende AV. Agricultura e qualidade da água: contaminação da água por nitrato. Planaltina: Embrapa Cerrados; 2002.

Rodrigues VA. Morfometria e mata ciliar da microbacia hidrográfica. In: Rodrigues VA, Starzynski $\mathrm{R}$, organizadores. Workshop em manejo de bacias hidrográficas. Botucatu: FEPAF:FCA:DRN; 2004.

Rodrigues VA. Recuperação de nascentes em microbacias da cuesta de Botucatu. In: Rodrigues
VA, Bucci LA, organizadores. Manejo de microbacias hidrográficas: experiências nacionais e internacionais. Botucatu: FEPAF; 2006.

Silva FAZ, Azevedo CAV. Versão do programa computacional Assistat para o sistema operacional Windows. Revista Brasileira de Produtos Agroindustrais 2002; 4(1): 71-78.

Silva KC. Qualidade da água ao longo do rio Capivara no município de Botucatu - SP [dissertação]. Botucatu: Faculdade de Ciências Agronômicas, Universidade Estadual Paulista; 2007.

Silva PE. A influência da mata ciliar na qualidade da água em dois rios na área rural de Foz do Iguaçu - PR [monografia]. Foz do Iguaçu: Faculdade Dinâmica de Cataratas, União Dinâmica de Faculdade Cataratas; 2009.

Simões LB. Integração entre um modelo de simulação hidrológica e sistema de informação geográfica na delimitação de zonas tampão ripárias [tese]. Botucatu: Faculdade de Ciências Agronômicas, Universidade Estadual Paulista; 2001. 